\title{
Numerical simulation and modeling of droplet spreading under smaller Weber numbers
}

Ning Wang ${ }^{1}$, Changlu Zhao ${ }^{1}$, Zhenyu Zhang*1

${ }^{1}$ School of Mechanical Engineering, Beijing Institute of Technology, Beijing, China

${ }^{*}$ Corresponding author email: zhenyu.zhang@bit.edu.cn

\begin{abstract}
Dynamic of droplet spreading on the free-slip surface was studied numerically by using the Front tracking method (FTM), with particularly interesting in the impacting under relatively small droplet inertias $(W e \leq 30)$. Our predictions of dimensionless droplet maximum spreading diameter $\beta_{\max }$ agree well with the widely-used Wildeman et al.'s [J. Fluid Mech. 805: 636-655 (2016)] model at We>30. The "1/2 rule" (i.e., approximately one half of the initial kinetic energy finally transfer into surface energy) was found to break down at small Weber numbers (We $\leq$ 30) and droplet height is non-negligible when the energy conservation approach is employed to estimate $\beta_{\text {max }}$. Droplet spreading can be divided into three distinct regimes according to the deformation styles, namely, the puddle-shaped regime (I), the transition regime (II) and the pizza-shaped regime (III). Surface energy dominates the energy budget in regime (I), while kinetic energy dominates the energy budget in regime (III). A practical model for estimating $\beta$ _max under small Weber numbers $(\mathrm{We} \leqslant 30)$ was proposed by accounting for the influence of impact parameters on the energy budget and the droplet height. Good agreement was found between our model and previous experiments.
\end{abstract}

\section{Keywords}

Droplet spreading; Small Weber numbers; Maximum spreading diameter; Modeling.

\section{Introduction}

Droplet impact a solid surface is regularly observed in many nature and industrial process [1].As for direct-ignition engines, liquid fuel is more likely to impact the combustion chamber due to the increasingly high fuel injection quantity [2]. Spray/wall interaction characteristics substantially influences mixture formation and subsequent combustion, therefore influences engine efficiency and emission. One of the most important underlying physics of spray/wall interaction is the dynamic of droplet impacting solid surface, which is usually adopted to estimate the wall film performance [3].

As a fundamental phenomenon in investigating spray/wall interaction, dynamic of droplet impacting the solid surface has been studied for decades. Previous investigations indicate that impacting dynamic is controlled by the impact Weber number $W e=\rho_{l} D_{0} U_{0}^{2} / \sigma_{l}$, which measures the relative importance of droplet inertia with respect to its surface tension; the Reynolds number $R e=\rho_{l} D_{0} U_{0} / \mu_{l}$, characterizing the relationship between droplet inertia force and its viscous force, where $D_{0}$ is the droplet initial diameter, $U_{0}$ the impact velocity, $\rho_{l}$, $\mu_{l}, \sigma_{l}$, are the droplet density, viscosity and surface tension, respectively. For droplet spreading, one of the most important factors to estimate droplet dynamic is the dimensionless droplet maximum spreading diameter $\beta_{\max }$, which is defined as the ratio of droplet maximum spreading diameter $D_{m}$ over its initial diameter $D_{0}$, yields $\beta_{\max }=D_{m} / D_{0}$. Quantifying $\beta_{\max }$ is useful in many practical applications, such as estimating oil film characteristics in different engines [3].

A considerable number of models have been proposed for $\beta_{\max }$ quantifying, which have been reviewed in some excellent literatures [4-6]. Normally, the existing models for predicting 
$\beta_{\text {max }}$ can be divided into three different categories according to the analysis methods, namely, scaling law momentum conservation and energy conservation. For viscous fluid such as liquid hydrocarbon fuel, the contribution of viscous dissipation on energy conservation is nonnegligible therefore energy conservation approach tends to predicted more accurate $\beta_{\max }$. In this case, accurately predicting the viscous dissipation rate hence dissipated kinetic energy is essential for predicting droplet dynamic characteristics.

To account for viscous dissipation during spreading, Wildeman et al. [7] numerically simulated droplet spreading under two limiting conditions, i.e., spreading on the total free-slip surface and no-slip surface. They proposed a "1/2 rule" that around one half of the kinetic energy finally transforms into surface energy for droplet spreading on an ideal free-slip surface under relatively large Weber numbers $(W e>30)$ and Reynolds numbers. The " $1 / 2$ rule" allows a complete theoretical solution for $\beta_{\max }$ prediction. The uniform droplet deformation under relatively large $W e$ may be the main reason for uniform kinetic energy dissipation and the asymptotically accurate "pizza-shaped" deformation for $W e>30$ indicates droplet inertia dominates droplet internal flow, which almost regardless of $R e$. In spite of these advances in understanding droplet spreading dynamic under large Weber numbers $(W e>30)$, it is surprising to find that only a few works have been conducted for small Weber numbers $(W e \leq$ 30 ). Most of the previous studies were focused on modeling $\beta_{\max }$ under large $W e$ where droplet deformation appears a uniform "pizza-shaped" styles hence energy dissipation is $W e-$ independent. However, modeling $\beta_{\max }$ under relatively small $W e$ has been barely investigated, probably due to the complex droplet deformation and energy dissipation characteristics.

Based on the discussion state above on, the present study aims to numerically investigate the impacting dynamics and model $\beta_{\max }$ under relatively small Weber numbers $(W e \leq 30)$. Following our previous numerical works [8-10], a well-validated Front tracking method (referred to as FTM hereinafter) was employed to conduct the numerical simulations. To eliminate the influence of extra factors such as gas film effects and surface properties, we only considered an ideal condition that droplet spread with a gas film under free-slip surface. In the following text, we shall first introduce our numerical approach in Section II, followed by the discussion of spreading dynamic, particularly for small Weber numbers. Subsequently, the correlation between impact parameter and $\beta_{\max }$ will be discussed. Final, the conclusion should be presented in the last section.

\section{Numerical Methodology}

The present numerical study was conducted by adopting the FTM, which is originally developed by Unverdi et al. [11] and Tryggvason et al. [12] To simulate droplet impact on a free-slip surface for incompressible two-phase flow. A second-order centered difference scheme for the spatial variables and an explicit first order time integration method were employed to solve the governing equations of both liquid and gas phases, given by

$$
\nabla \cdot \boldsymbol{U}=0
$$

$$
\frac{\partial(\rho \boldsymbol{U})}{\partial t}+\nabla \cdot(\rho \boldsymbol{U} \boldsymbol{U})=-\nabla p+\nabla \cdot \mu\left[\nabla \boldsymbol{U}+(\nabla \boldsymbol{U})^{T}\right]-\sigma \int \kappa \boldsymbol{n} \delta\left(\boldsymbol{x}-\boldsymbol{x}_{\boldsymbol{f}}\right) d A
$$

where $\boldsymbol{U}$ is the velocity vector, $\rho$ is the density, $p$ is the pressure, $\mu$ is the viscosity, $\sigma$ is the surface tension coefficient, $\kappa$ is twice the mean curvature of local field, $\boldsymbol{n}$ is the unit vector outwardly normal to the local front, the term $\delta$ is the constructed three-dimensional $\delta$ function which represents the singular force, surface tension, over the phase interface, $x$ is the point at which the equation is evaluated and $\boldsymbol{x}_{\boldsymbol{f}}$ is a point on the front. 
The FTM solves a single set of conservation equations with appropriate interface terms in the whole field with an Eulerian coordinates grid and tracks liquid-gas interfaces using a Lagrangian approach. Figure 1 shows the computational domain of the current numerical simulation. In the cylindrical coordinate, $z$-axis is structured by the droplet initial velocity normal to the surface, while $r$-axis is perpendicular to the droplet initial velocity. Axisymmetric boundary condition is specified for the $z$-axis, while free-slip boundary conditions are specified to all of the other boundaries including the impacting solid surface.

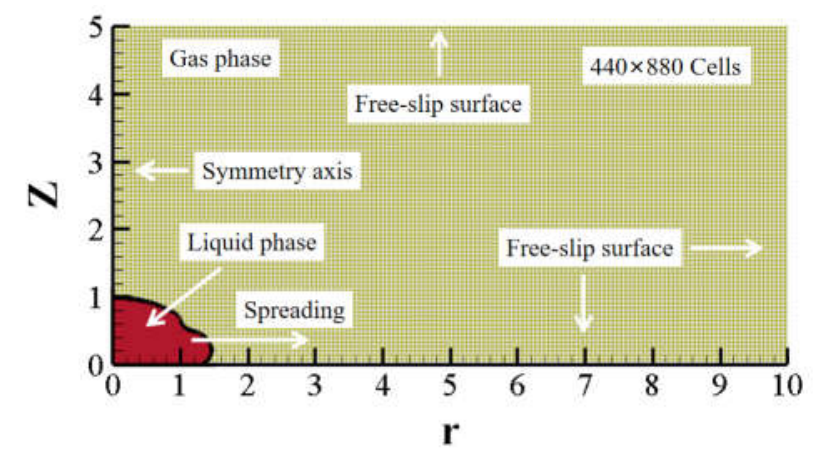

Figure 1. Axisymmetric computational domain with uniform structured grids.

The computational domain of width $10 R_{0}$ and height $5 R_{0}$ is discretized by a uniform orthogonal mesh with $440 \times 880$ cells, which means each unit length contains 88 grid points. Grid-dependence of present numerical approach have been fully checked in many previous works $[9,13]$ in which FTM is not sensitive to the grid size because liquid-gas interface is tracked by the Lagrangian particles rather than computational cell. Additionally, the present cell size has been validated in one of the author's previous publication [9], in which simulations with the same cell size have been proved to successfully and accurately solve the droplet problems. It should be noted that droplet impact problem always involving in the complex gas film problems. Therefore, to simplify the present simulation but avoid the complication of gas film, we only focus on a limiting condition that droplet spread with a gas film throughout the whole process.

Governing equations are nondimensionalized by the droplet radius $R_{0}=D_{0} / 2$, initial velocity $U_{0}$ and droplet density $\rho_{l}$. Time is normalized by using $T=t \cdot U_{0} / D_{0}$, where $t$ is the real time. In addition, several previous studies $[8,9]$ confirm that gas-liquid density ratio $\rho_{g} / \rho_{l}$ and viscosity ratio $\mu_{g} / \mu_{l}$ are two or three orders of magnitude smaller than unity. Therefore their effects on droplet deformation and viscous dissipation can be neglected. Where $\rho_{g}$ and $\mu_{g}$ are gas density and viscosity, respectively.

The FTM adopted in the present study has been sufficiently applied and validated in many previous studies [8-10] on droplet dynamic problems, which demonstrates that the present numerical method can be used to accurately predict the evolution of droplet deformation during impacting.

Considering spreading on the free-slip surface with a gas film is equivalent to the impacts of Leidenfrost droplets, which hover on a steam gas film. We simulated Tran et al.'s [14] experiments on Leidenfrost droplet spreading at $\mathrm{We}=32$ and $\mathrm{Re}=2150$, as shown in Figure 2. The simulation precisely predicted the droplet contours in the earlier stage by $t=1.2 \mathrm{~ms}$, beyond which it seems our prediction shows lower droplet center height. This observation can be understood by recognizing that droplet spreading is accurately a rotational symmetry problem. Nevertheless, good agreement between our prediction and previous experimental results in terms of instantaneous droplet spreading diameter demonstrates the present 
numerical approach can accurately predict the evolution of droplet spreading during impacting a free-slip surface with a gas film.

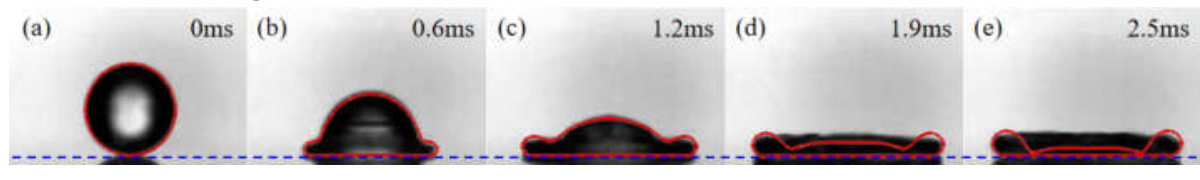

Figure 2. Time sequences of Leidenfrost droplet spreading at $\mathrm{We}=32$ and $\mathrm{Re}=2150$. The solid line denotes predicted results while the shadowgraph indicates experimental results of Tran et al. [14] ; Horizontal line for impacting surface is represented by blue dash line.

\section{Results and Discussion}

\subsection{Droplet spreading under relatively large Weber numbers $(W e>30)$}

Energy conservation approach where the initial kinetic energy $E_{k 0}$ is equal to the sum of dissipation energy $E_{d}$, left-over kinetic energy $E_{k}$ and surface energy increment $\Delta E_{s}$ at max deformation instant $\tau_{m}$, yields

$E_{k 0}=E_{d}+E_{k}+\Delta E_{s}$

Since Wildeman et al.'s model is estimated according to the energy conservation approach associate with the "1/2 rule", we subsequently show the energy budget in Figure 3 , in which all the energies were normalized by the initial kinetic energy $E_{k 0}$ and therefore the sum of $E_{k}, E_{d}$ and $\Delta E_{s}$ is the unity. To further investigate the viscous dissipation characteristics, we counted the energy dissipation in both liquid phase (denoted by $E_{d l}$ ) and gas phase (denoted by $E_{d g}$ ), and therefore $E_{d}=E_{d l}+E_{d g}$. Figure 3(a) shows the evolution of four different energies during droplet spreading process, while Figure 3(b) shows the energy budget at $\tau_{m}$ for various droplet inertias and Reynolds numbers. In this case we defined $\tau=0$ as the time instant when the droplet just contracts the surface. The initially non-zero $E_{d}$ at $\tau=$ 0 , shown in Figure 3(a), can be attributed to the slightly deformation together with the droplet moving-induced gas motion and slightly influences the energy budget, therefore can be ignored in the present study. For energy budget shown in Figure 3(b), all the predictions generally follow the " $1 / 2$ rule". Although viscous dissipation in the liquid phase contributes more for $E_{d}$, viscous dissipation in the gas phase, i.e., $E_{d g}$ still cannot be ignored.

\subsection{Droplet spreading under relatively small Weber numbers (We $\leq \mathbf{3 0}$ )}

Figure 4 shows the prediction of $\beta_{\max }$ under small Weber numbers $(W e \leq 30)$. The solid line indicates Wildeman et al.'s [7] model. It is seen that $\beta_{\max }$ slightly deviates from Wildeman et al.'s model as droplet inertia decreases from $W e=30$ to around $W e=12$. This deflection can be attributed to the breakdown of the " $1 / 2$ rule" and the dominant factor of energy budget transfer from kinetic energy to the surface energy, to be elucidated in the following text. For Wildeman et al.'s model, there are two important hypotheses i.e., around one half of the initial kinetic energy transfers into surface energy and droplet shape at $\tau_{m}$ can be regarded as a "pizza-shaped" disk, whose thickness can be ignored when compared to the spreading diameter.

To investigate the reason why droplet spreading under small Weber numbers deviates from the Wildeman et al.'s model, we show the energy budget for $W e \leq 30$ in Figure 5. It is clearly seen that the " $1 / 2$ rule" breaks down and $\Delta E_{s}$ increases with Weber number decreasing, indicating an $W e$-dependent energy budget. $\Delta E_{S}$ generally larger than $1 / 2$ indicates surface energy may be play a dominant role on droplet deformation during droplet spreading [8]. 

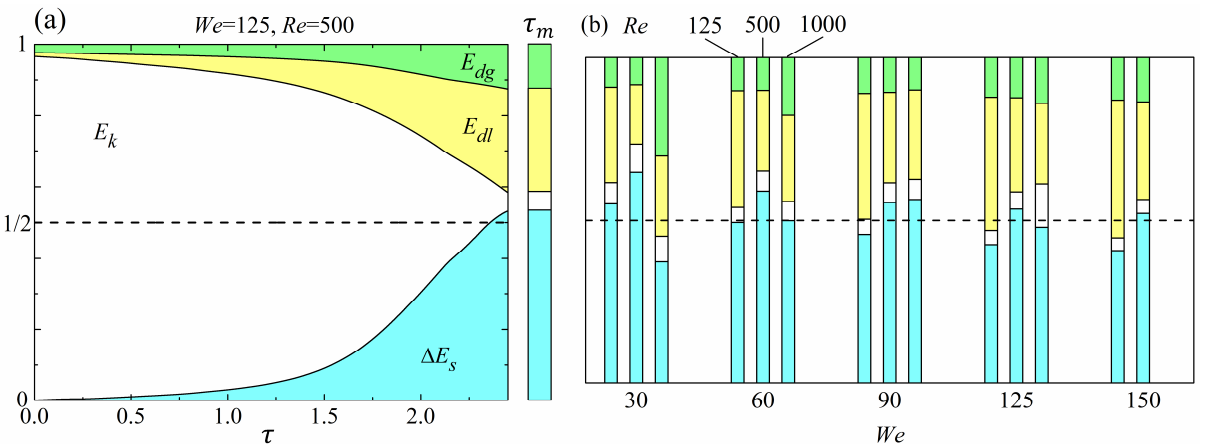

Figure 3. Energy budget of droplet spread on the free-slip surface under $\mathrm{We} \geqslant 30$. The dash line indicates the “ $1 / 2$ rule”.

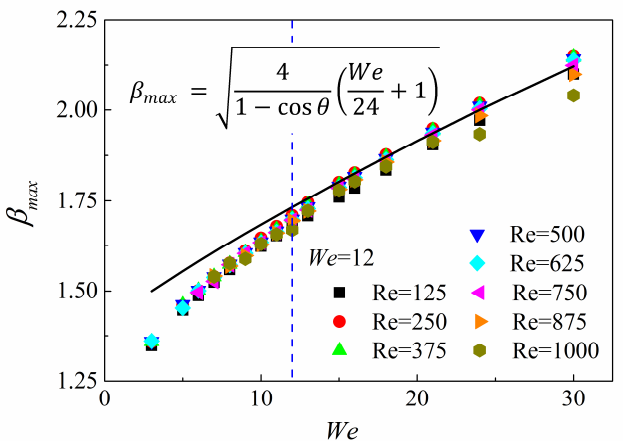

Figure 4. $\beta_{\max }$ under small Weber numbers $(\mathrm{We} \leqslant 30)$. The Wildeman et al.' $\mathrm{s}[7]$ model is indicating in the figure for a comparison.

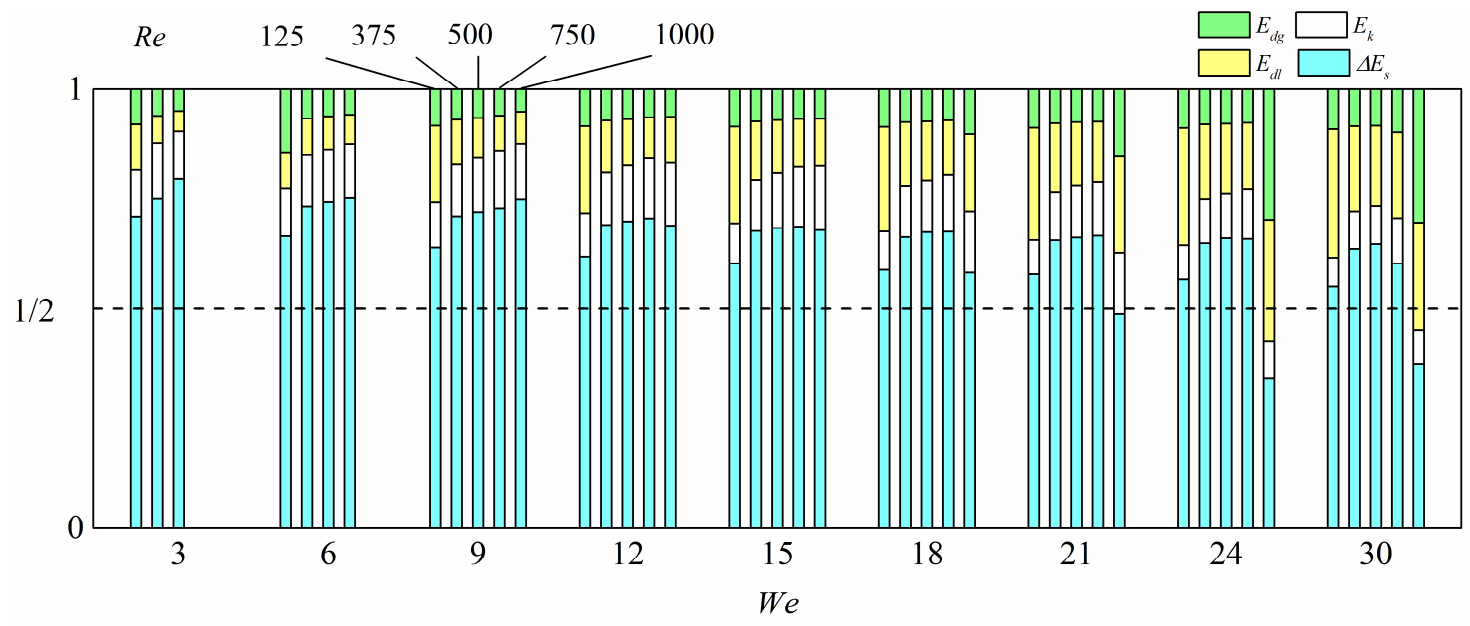

Figure 5. Energy budget under small Weber numbers for different Reynolds numbers.

\subsection{Droplet deformation transitions}

Another significant factor should be taken into account is the non-negligible droplet height under small Weber numbers $(W e \leq 30)$. In this study we employed three different heights to characterize droplet shape, namely, droplet center height $h_{c}$, throat height $h_{t}$ and rim height $h_{r}$. Figure 6(a) shows the evolution of three heights with impact Weber number increasing, the Reynolds number was fixed at $R e=1000$. Droplet deformation shows a complex tendency and can be divided into three distinct regimes as impact Weber number increases, namely, puddle-shaped regime (I), transition regime (II) and pizza-shaped regime (III).

For relatively smaller Weber number $(W e<12)$ in regime $(I)$, it seems $h_{c}$ shows the highest value among three heights while $h_{t}$ shows the lowest ones, indicates that relatively 
small impact inertia results in that droplet appears like a puddle. As droplet inertia increases to regime (II), the initial kinetic energy gradually dominates the energy transfer therefore a rim can be squeezed out from the bottom part of the droplet and a narrow neck throat is formed to connect the rim and the central part of the droplet. As droplet impact inertia continually increases and finally beyond $W e=30$, i.e., regime (III), we found $h_{c}$ is approach to the $h_{t}$ and its evolution becomes steady, indicates that droplet deformation become more pronounced and a thin lamella is bordered by a rim therefore droplet height can be ignored when estimating the surface energy.

To further show the transition of droplet deformation, we quantitatively compared the relationship between droplet center height $h_{c}$ and the rim height $h_{r}$ denoted as $\Delta h=h_{c}-h_{r}$, as shown in Figure 6(b). When the droplet spreading in regime (I), i.e., $W e<12$, initial kinetic energy is insufficient strong to overcome the constraints of surface tension hence $\Delta h$ generally larger than zero. As droplet inertia increases into regime (II), $\Delta h$ decreases linearly by $W e=$ 30 , beyond which droplet spread in the regime (III) and $\Delta h$ tends to become steady, indicates a uniform droplet deformation where droplet height is negligible and associating with the " $1 / 2$ rule", $\beta_{\max }$ can be accurately estimated according to energy conservation. However, as $W e$ decreases to regime (II) $(12 \leq W e \leq 30)$ although the "1/2 rule" breaks down, the pizzashaped droplet reluctantly maintains, therefore $\beta_{\max }$ only slightly deviates from Wildeman et al.'s model. As $W e$ further decreases to $W e<12$, droplet deformation transition into puddleshape regime (I) where droplet height is non-negligible when estimate $\beta_{\text {max }}$, therefore produces substantial deflection.

(a)

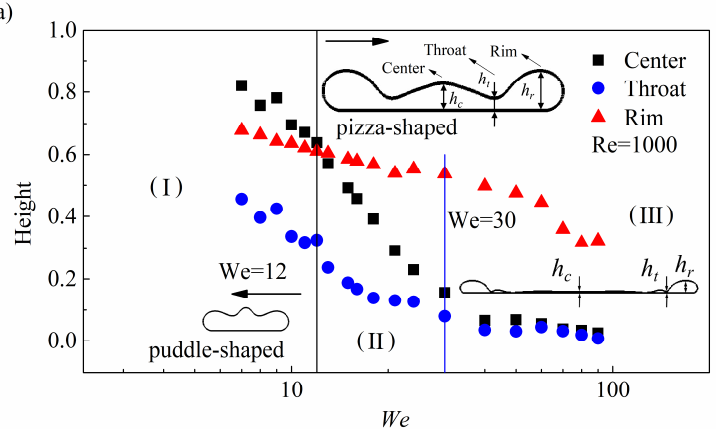

(b)

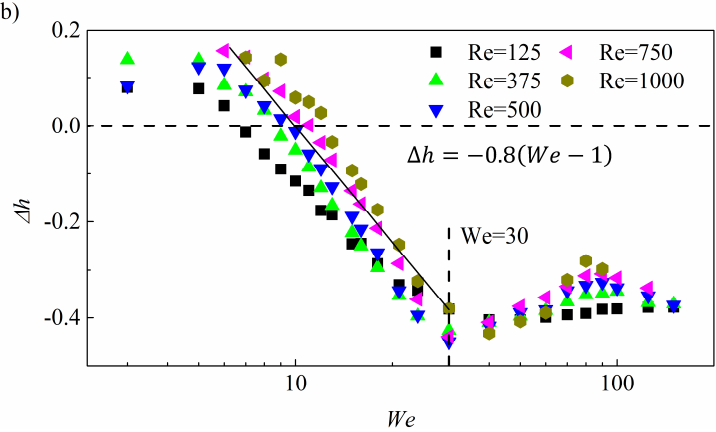

Figure 1. Schematic of droplet deformation transition characterized by different heights.

\subsection{Modeling $\boldsymbol{\beta}_{\max }$ under small Weber numbers}

It has been recognized in the previous section that the "1/2 rule" breaks down when the droplet impact inertia decreases to $W e \leq 30$, therefore $\beta_{\max }$ gradually deviates from the Wildeman et al.'s model. In this section, we first adopted a revised Weber number $W e_{r}$, which is defined as the droplet initial kinetic energy $\left(E_{k 0}=\pi \rho V_{0}^{2} D_{0}^{3} / 12\right)$ over its initial surface energy $\left(E_{s 0}=\sigma \pi D_{0}^{2}\right)$ hence $W e_{r}=W e / 12$. The $W e_{r}$ has been employed by Zhang and Zhang [8] to precisely measure the relative importance between droplet initial kinetic energy and surface energy.

To estimate $\beta_{\max }$ using energy conservation approach, we first introduced the droplet deformation ratio $R_{d}$ as the rate of surface energy increment $\Delta E_{s}$ to initial kinetic energy $E_{k 0}$, given by $R_{d}=\Delta E_{s} / E_{k 0}$. Figure 7 is used to show the relationship between $R_{d}$ and droplet revised Weber number $W e_{r}$, surface energy increment can be express as $\Delta E_{s}=R_{d} \cdot E_{k 0}$.

Regardless of droplet spreading under relatively large $R e$ cases for $W e_{r}>1.0, R_{d}$ seems independent of the $R e$ and the simulation results agree well with the fitted formula, given by $R_{d}=0.7 W e_{r}^{-0.1}$ 
Since droplet height is non-negligible when estimating surface energy under small Weber numbers $(W e \leq 30)$, droplet deformation and its transition can be characterized by three distinct heights, namely, the center height $h_{c}$, the throat height $h_{t}$ and the rim height $h_{r}$. To simplify the quantification on surface energy, droplet at $\tau_{m}$ can be equivalent to a "columnshaped" cylinder with the identical droplet maximum spreading diameter $D_{m}$ and surface energy, as shown in Figure 8(a). Therefore, droplet height characterization can be simplified by using an equivalent surface energy height $h_{e}$, in this case surface energy can be expressed as

$$
E_{s}\left(\tau_{m}\right)=\sigma \pi\left[D_{m} h_{e}+\frac{D_{m}^{2}(1-\cos \theta)}{4}\right]
$$

where $\theta$ is the contract angle and can be determined as $180^{\circ}$ in our simulations. $h_{e}$ can be further normalized by the $D_{m}$ as $R_{e}=h_{e} / D_{m}$, which measures the relative importance of droplet height when compared to the droplet spreading diameter, and we found that $R_{e}$ is relate to droplet impact inertia, as shown in Figure 8(b) that,

$$
R_{e}=0.1 W e_{r}^{-0.4}
$$

Consequently, $\beta_{\max }$ can be estimated by accounting for droplet surface energy increment at $\tau_{m}$, yields

$$
\left.\beta_{\text {max }}=\sqrt{\frac{0.7 W e_{r}^{0.9}+0.1}{0.1 W e_{r}^{-0.4}+(1-\cos \theta) / 4}} \text { (Free-slip, } W e \leq 30\right)
$$

Figure 9 shows the comparison between the present model (i.e., equation 7, denotes by the red line) and the available numerical and experimental results. Serval observations can be made in this figure as follows. Firstly, our numerical predictions agree well with Tran et al.'s [14] experimental data which again confirms the accuracy of the present simulation. Secondary, the derived model for estimating $\beta_{\max }$ under small Weber numbers $(W e \leq 30)$ shows good agreement with both Tran et al.'s experimental results and our numerical predictions. Finally, the present model and Wildeman et al.'s model shows fine continuity at $W e=30$.

\section{Conclusions}

(1) As droplet impact inertia decreases to $W e \leq 30$, where a $W e$-dependent energy budget can be found therefore the " $1 / 2$ rule" breaks down. Droplet progressively loss its thin pizzalike shape and finally degenerates into puddle-like shape whose height is non-negligible when estimating the surface energy.

(2) Droplet spreading can be divided into three distinct regimes. The first regime (I) is characterized by the puddle-shaped droplet at $\tau_{m}$, in this case droplet surface energy dominates energy budget and restricts droplet deformation. The second regime (II) is regarded as the transition regime. In this case, initial kinetic energy is comparable to the surface energy. The third regime (III) is characterized by the uniform droplet deformation (thin pizza-shaped), where the " $1 / 2$ rule" is validated and droplet height is negligible when compared to the spreading diameter.

(3) Based on the understanding of droplet spreading under small Weber numbers $(W e \leq 30)$, energy conservation approach was again employed to model the dimensionless droplet maximum spreading diameter $\beta_{\max }$ by accounting for the influence of impact parameters (such as $W e$ and $R e$ ) on the energy budget and the droplet height at $\tau_{m}$. A revised Weber number $W e_{r}$, which correctly reflects the orders of magnitude of various energies, was adopted to replace the $W e$ in our modeling works. 


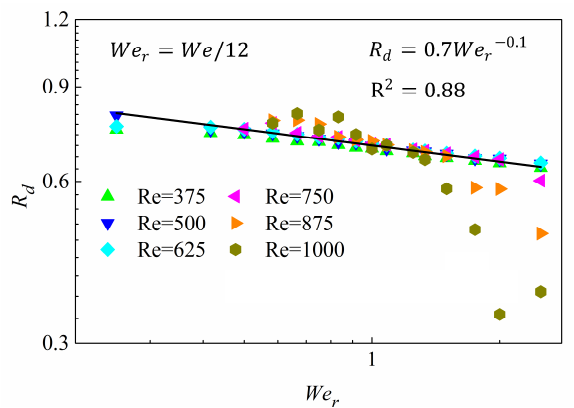

Figure 7. Correlation between $R_{d}=$ $\Delta E_{s} / E_{k 0}$ and revised Weber number with $\mathrm{R}^{\wedge} 2=0.88$.
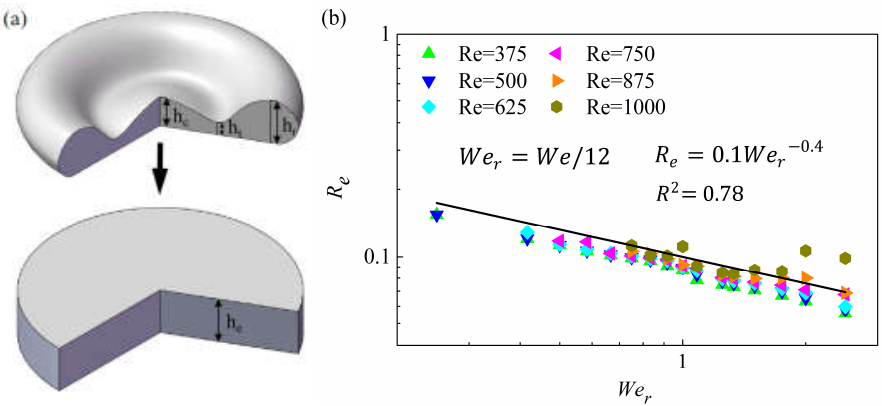

Figure 8. (a) Schematic of equivalent droplet deformation and (b) correlation between the normalized droplet equivalent height $R_{e}=$ $h_{e} / D_{m}$ and revised Weber number $W e_{r}$ with $R^{2}=0.78$.

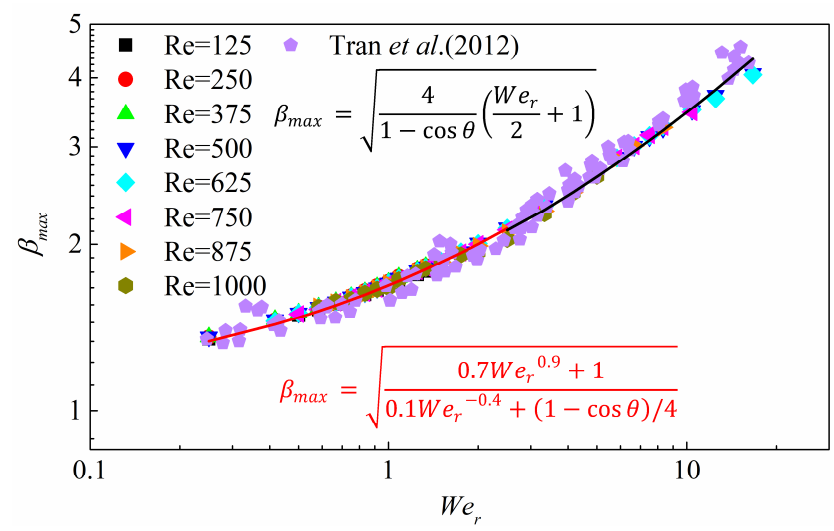

Figure 9. Comparison between the derived spreading models (black solid line indicates Wildeman et al.'s [7] model, while red line denotes the present proposed model) and data points (Tran et al.'s experiment and numerical predictions.

\section{Acknowledgment}

The authors would like to acknowledge the research grant received from National Natural Science Foundation of China (grant no. 51806013), Foundation research funds of Ministry of Industry and Information Technology (grant no. JCKY2019602D018) and Beijing Institute of Technology Research Fund Program for Young Scholars (grant no. 2020CX04047).

\section{References}

[1] Yarin, A. L., 2006, Annu. Rev. Fluid Mech, 38 (1), pp. 159-192.

[2] Tang, C., Mengxiao, Q., Xinyan, W., 2017, Int. J. Multiphase Flow, 96, pp. 56-69.

[3] Moreira, A.L.N., Moita, A. S., Panão, M.R., 2010, Energy Combust. Sci, 36 (5), pp. 554580.

[4] Bennett, T., Poulikakos, D., 1993, J. Mater. Sci., 28 (4), pp. 963-970.

[5] German, G., Bertola, V., 2009, Atom. Sprays, 19 (8), pp. 787-807.

[6] Marengo, M., Antonini, C., 2011, Curr. Opin. Colloid Interface Sci., 16 (4), pp. 292-302.

[7] Wildeman, S., Visser, C. W., 2016, Fluid Mech, 805, pp. 636-655.

[8] Zhang, Z., Zhang, P., 2019, Langmuir., 35 (49), pp. 16164-16171.

[9] Zhang, Z., Zhang, P., 2017, Phys. Fluids, 29 (10), pp. 103306.

[10] Zhang, Z., Zhang, P., 2018, Atom. Sprays, 28 (8), pp. 673-694.

[11] Unverdi, S. O., Tryggvason, G., 1992, J. Comput. Phys., 100 (1), pp. 25-37.

[12] Tryggvason, G., et al., 2001, J. Comput. Phys., 169 (2), pp. 708-759.

[13] Nobari, M.R.H., Jan, Y. J., 1993, Phys. Fluids, 8 (1), pp. 29.

[14] Tran, T., Staat, H.J.J., 2012, Phys. Rev. Lett., 108 (3), pp. 036101. 